\title{
Static critical behavior of the two-dimensional Ising spin glass $\mathrm{Rb}_{2} \mathrm{Cu}_{1-x} \mathrm{Co}_{x} \mathrm{~F}_{4}$
}

\author{
C. Dekker, A. F. M. Arts, and H. W. de Wijn \\ Fysisch Laboratorium, Rijksuniversiteit Utrecht, Postbox 80000, 3508 TA Utrecht, The Netherlands
}

(Received 20 May 1988)

The temperature and field dependences of the nonlinear susceptibility are examined in $\mathrm{Rb}_{2} \mathrm{Cu}_{1-x} \mathrm{Co}_{x} \mathrm{~F}_{4}$ with $x=0.218$, which is an experimental realization of the two-dimensional Edwards-Anderson Ising spin glass with random nearest-neighbor bonds. From the divergence of the nonlinear terms in the expansion of the magnetization in the field as well as from static scaling, the spin-glass transition is shown to occur at zero temperature, which implies the lower critical dimensionality for Ising spin glasses to exceed 2. The critical exponents $\gamma=4.5 \pm 0.2$ and $\beta=0.0 \pm 0.1$ agree with recent theoretical estimates for the $\pm J$ Ising spin glass in two dimensions.

\section{INTRODUCTION}

In the last few years, major progress has been made in elucidating whether the peculiar characteristics of spin glasses $^{1,2}$ (SG) indicate an equilibrium phase transition. In particular, experiments on the singular behavior of the nonlinear susceptibility have played a decisive role in providing evidence for a transition at a finite critical temperature $T_{c}$. In the framework of the mean-field theory, ${ }^{3}$ the nonlinear susceptibility had earlier been shown to scale with the SG order-parameter susceptibility, ${ }^{4,5}$ and therefore to diverge according to a power law. Such a critical behavior has at present been established in a variety of SG compounds, all of which are three-dimensional $(d=3) .^{6-9}$ In the case of $d=2$ systems, by contrast, theoretical arguments inclusive of extensive numerical simulations have inferred that $T_{c}=0$, despite a freezing at a finite temperature. ${ }^{10-17}$ Experimental verification of this important result however, is lacking.

In this paper, we investigate the temperature and field dependences of the nonlinear susceptibility in the shortrange $d=2$ Ising $S G \mathrm{Rb}_{2} \mathrm{Cu}_{0.782} \mathrm{Co}_{0.218} \mathrm{~F}_{4}$, ${ }^{18}$ with the primary aim to determine whether $T_{c}$ is zero or finite from the static critical behavior. The results evidence a power-law divergence of the leading nonlinear terms in an expansion of the susceptibility at zero temperature, culminating in an excellent static $T_{c}=0$ scaling of the total nonlinear susceptibility. This implies that the lower critical dimensionality exceeds 2 . The critical exponents $\gamma$ and $\beta$ further appear to compare to theoretical estimates.

\section{MAGNETIC PROPERTIES OF $\mathrm{Rb}_{2} \mathrm{Cu}_{1-x} \mathrm{Co}_{x} \mathrm{~F}_{4}$}

In this section, we discuss the magnetic properties of $\mathrm{Rb}_{2} \mathrm{Cu}_{1-x} \mathrm{Co}_{x} \mathrm{~F}_{4}$ in relation to the $d=2$ Ising EdwardsAnderson model for short-range SG. ${ }^{19}$ The crystal structure of $\mathrm{Rb}_{2} \mathrm{Cu}_{1-x} \mathrm{Co}_{x} \mathrm{~F}_{4}$ is, as that of the pure end members, of the $\mathrm{K}_{2} \mathrm{NiF}_{4}$ type. The magnetic ions are situated on a simple square lattice within widely separated $\mathrm{Cu}_{1-x} \mathrm{Co}_{x} \mathrm{~F}_{2}$ layers. The intralayer exchange is at least 3 orders of magnitude stronger than the interlayer exchange. $\mathrm{Rb}_{2} \mathrm{CoF}_{4}$ is an archetypal $d=2$ Ising antiferromagnet, with the spins ordering along the tetragonal axis below a Néel temperature $T_{N}=103.0 \mathrm{~K} .^{20,21}$ $\mathrm{Rb}_{2} \mathrm{CuF}_{4}$ is a $d=2$ ferromagnet with predominant Heisenberg exchange and small $(\sim 1 \%) X Y$ anisotropy leaving the spins within the plane. ${ }^{22}$ The Curie temperature is $T_{c}=6.05 \pm 0.09 \mathrm{~K}$. The magnetic interactions in $\mathrm{Rb}_{2} \mathrm{Cu}_{1-x} \mathrm{Co}_{x} \mathrm{~F}_{4}$, primarily originating from exchange, are to a high degree between nearest neighbors only, with next-nearest-neighbor interactions down to $\sim 1 \%$.

Randomizing $\mathrm{Co}$ and $\mathrm{Cu}$ spins over the square lattice results in a distribution of interactions that simulates bond randomness. In $\mathrm{Cu}$ compounds of the $\mathrm{K}_{2} \mathrm{NiF}_{4}$ structure, the $\mathrm{Cu}^{2+} d_{z^{2}-x^{2}}$ and $d_{z^{2}-y^{2}}$ ground-state orbitals are arranged such that they alternate along the crystallographic [100] and [010] axes, leading to a ferromagnetic $\mathrm{Cu}-\mathrm{Cu}$ exchange. ${ }^{23}$ As illustrated in Fig. 1(a), substitution of $\mathrm{Co}$ for $\mathrm{Cu}$ results in two distinct $\mathrm{Cu}-\mathrm{Co}$ interactions, dependent on the orientation of the $\mathrm{Cu}^{2+}$ orbital relative to the $\mathrm{Cu}-\mathrm{Co}$ bond. The exchange is antiferromagnetic in case the $d$-orbital lobe points towards the $\mathrm{Co}^{2+}$ ion, and ferromagnetic otherwise. ${ }^{24}$ In $\mathrm{Rb}_{2} \mathrm{Cu}_{1-x} \mathrm{Co}_{x} \mathrm{~F}_{4}$, we thus have two ferromagnetic $(\mathrm{Cu}$ $\mathrm{Cu}$ and $\mathrm{Cu}-\mathrm{Co}$ ) and two antiferromagnetic interactions (Cu-Co and $\mathrm{Co}-\mathrm{Co}$ ). Both $\mathrm{Cu}$ and $\mathrm{Co}$ have, the latter effectively, a spin value $S=\frac{1}{2}$. The nearest-neighbor bond strength thus is the only random variable of relevance, making $\mathrm{Rb}_{2} \mathrm{Cu}_{1-x} \mathrm{Co}_{x} \mathrm{~F}_{4}$ an almost ideal representative of a $d=2$ random-bond magnetic system. The huge uniaxial crystalline anisotropy of the $\mathrm{Co}^{2+}$ ion further makes the $\mathrm{Co}-\mathrm{Co}$ and the two $\mathrm{Cu}-\mathrm{Co}$ interactions strongly Ising-like in character. As it turns out, SG freezing is achieved for $0.18 \leq x \leq 0.40 .{ }^{22}$ Summing up, therefore, $\mathrm{Rb}_{2} \mathrm{Cu}_{1-x} \mathrm{Co}_{x} \mathrm{~F}_{4}$ constitutes an ideal realization of the $d=2$ Ising Edwards-Anderson model, except for minor details in the bond distribution.

In more detail, the explicit form of the bond probability $P(J)$ is, on the assumption of site randomness, given by

$$
\begin{aligned}
P(J)= & x^{2} \delta\left(J-J_{\mathrm{Co}-\mathrm{Co}_{0}}\right)+x(1-x) \delta\left(J-J_{\mathrm{Cu}-\mathrm{Co}_{0}}^{\mathrm{AF}}\right) \\
& +x(1-x) \delta\left(J-J_{\mathrm{Cu}-\mathrm{Co}_{0}}^{F}\right) \\
& +(1-x)^{2} \delta\left(J-J_{\mathrm{Cu}-\mathrm{Cu}_{\mathrm{u}}}\right)
\end{aligned}
$$

where $J_{A-B}$ is the exchange constant between $A$ and $B$, 
(a)

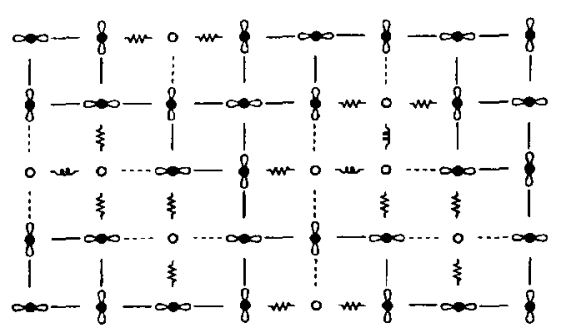

$$
\begin{aligned}
& \circ \mathrm{Co} \\
& -\mathrm{Cu} \\
& \text { AF o } \\
& \infty \mathrm{AF} \text {. } \\
& 8 \mathrm{~F} \text {. } \\
& 8 \text { F }
\end{aligned}
$$

(b)

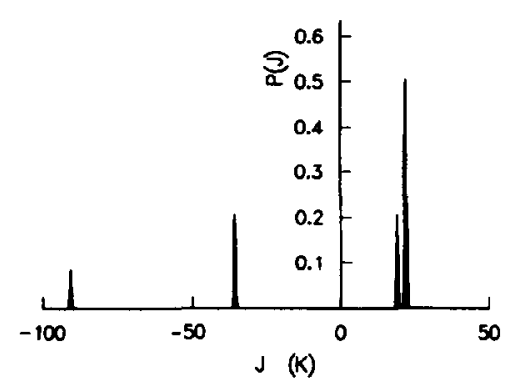

FIG. 1. (a) Representative distribution of ferromagnetic ( $F$ ) $\mathrm{Cu}-\mathrm{Cu}$ and $\mathrm{Cu}-\mathrm{Co}$ and antiferromagnetic (AF) $\mathrm{Co}-\mathrm{Co}$ and $\mathrm{Cu}$ Co exchange interactions in $\mathrm{Rb}_{2} \mathrm{Cu}_{1-x} \mathrm{Co}_{x} \mathrm{~F}_{4}$ for $x=0.22$. Also indicated are the in-layer $d$-orbital lobes of $\mathrm{Cu}^{2+}$. (b) Schematic bond probability function $P(J)$ according to Eq. (1) for $\mathrm{Rb}_{2} \mathrm{Cu}_{1-x} \mathrm{Co}_{x} \mathrm{~F}_{4}$ with $x=0.29$.

and the superscripts $\mathrm{AF}$ and $\boldsymbol{F}$ distinguish between the antiferromagnetic and ferromagnetic $\mathrm{Cu}-\mathrm{Co}$ bonds, respectively. We have $J_{\mathrm{Co} . \mathrm{Co}_{\mathrm{o}}}=-90.8 \mathrm{~K}$, as derived from $T_{N}$ in $\mathrm{Rb}_{2} \mathrm{CoF}_{4}$ with Onsager's rigorous result ${ }^{25}$ for the $d=2$ Ising system, and $J_{\mathrm{Cu} \cdot \mathrm{Cu}}=22.0 \mathrm{~K}$, as estimated from $J_{\mathrm{Cu}-\mathrm{Cu}}$ in $\mathrm{K}_{2} \mathrm{CuF}_{4}$ (Ref. 26) scaled with the Curie temperatures of the $\mathrm{Rb}$ and $\mathrm{K}$ compounds. To assess the $\mathrm{Co}-\mathrm{Cu}$ interactions, we make the reasonable assumption that at the midpoint of the SG range of concentrations, i.e., $x=0.29$, the average bond strength $\bar{J}=0$. By use of Eq. (1) and

$$
\bar{J}=\sum_{i=1}^{4} P\left(J_{i}\right) J_{i}
$$

with $i=1,2,3$, and 4 indexing the four bonds, it then follows that $J_{\mathrm{Cu}-\mathrm{Co}_{0}}^{\mathrm{AF}}+J_{\mathrm{Cu}-\mathrm{Co}_{0}}^{F}=-17 \mathrm{~K}$. From this in relation to $J_{\mathrm{Co}-\mathrm{Co}}$ and $J_{\mathrm{Cu}-\mathrm{Cu}}$, we estimate $J_{\mathrm{Cu}-\mathrm{Co}}^{F}=20 \pm 10 \mathrm{~K}$ and $J_{\mathrm{Cu}-\mathrm{Co}}^{\mathrm{AF}}=-37 \pm 10 \mathrm{~K}$. The resultant bond distribution is sketched in Fig. 1(b). Its discrete nature indicates that $\mathrm{Rb}_{2} \mathrm{Cu}_{1-x} \mathrm{Co}_{x} \mathrm{~F}_{4}$ compares to the $\pm J$ Edwards-Anderson SG model, which has

$$
P\left(J^{\prime}\right)=\frac{1}{2} \delta\left(J^{\prime}-J\right)+\frac{1}{2} \delta\left(J^{\prime}+J\right),
$$

better than to the Gaussian counterpart, which is characterized by a continuous distribution of $J$, symmetric about zero. An additional argument in favor of the $\pm J$ model is provided by the nonvanishing number of spins in $\mathrm{Rb}_{2} \mathrm{Cu}_{1-x} \mathrm{Co}_{x} \mathrm{~F}_{4}$ that can be flipped without any energy cost because of completely balancing exchange fields. ${ }^{22}$ Such spins are indeed abundant in the $\pm J$ model, but virtually absent in the Gaussian model. Although in the case of $d=2$ both models have $T_{c}=0$, the critical exponents differ. The models thus seem to represent different universality classes, ${ }^{13,16}$ presumably because the Gaussian model has a nondegenerate ground state for $T_{c}=0$, whereas the $\pm J$ model has a finite ground-state entropy.

The typical spread of the distribution of $J$ in $\mathrm{Rb}_{2} \mathrm{Cu}_{1-x} \mathrm{Co}_{x} \mathrm{~F}_{4}$, defined by the second moment

$$
\Delta J=\left[\sum_{i=1}^{4} P\left(J_{i}\right)\left(J_{i}-\bar{J}\right)^{2}\right]^{1 / 2},
$$

is, together with $\bar{J}$, shown versus $x$ in Fig. 2 . It is seen that $\Delta J$ is as large as $30-40 \mathrm{~K}$ in the SG regime. The fact that SG freezing is only observed near $3 \mathrm{~K}$ $(\approx 0.1 \Delta J)$ thus is in itself already indicative of a zero critical temperature for the present $d=2$ SG model compound. In the mean-field theory of $S G,{ }^{3}$ a $S G$ phase is found for $|\bar{J}|<\Delta J$, whereas for $\bar{J}>\Delta J$ the system orders ferromagnetically, and similarly antiferromagnetically for $\bar{J}<-\Delta J$. From the inset of Fig. 2, which shows the ratio $\Delta J /|\bar{J}|$ as calculated from Eqs. (2) and (3), it is seen that the boundaries of the SG regime actually found in $\mathrm{Rb}_{2} \mathrm{Cu}_{1-x} \mathrm{Co}_{x} \mathrm{~F}_{4}, x=0.18 \pm 0.02$ and $0.40 \pm 0.01$, correspond to $\Delta J /|\bar{J}| \approx 3$.

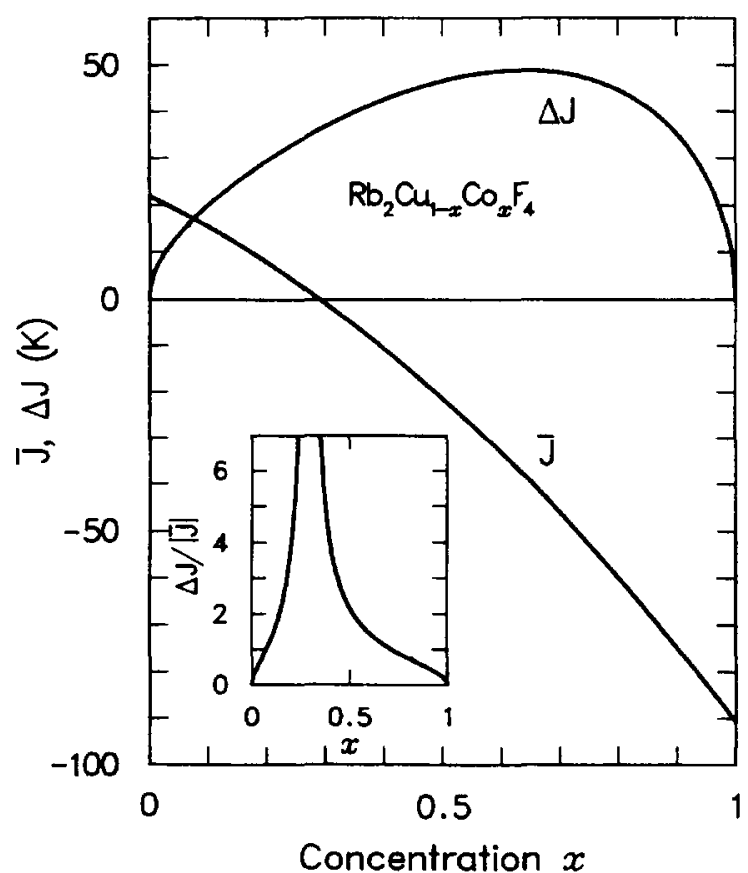

FIG. 2. Average bond strength $\bar{J}$ and the spread $\Delta J$ vs the concentration $x$ for $\mathrm{Rb}_{2} \mathrm{Cu}_{1-x} \mathrm{Co}_{x} \mathrm{~F}_{4}$. Inset shows $\Delta J /|\bar{J}|$ vs 


\section{EXPERIMENTAL DETAILS AND RESULTS}

The experiments were performed on a good-quality Czochralski-grown single crystal of $\mathrm{Rb}_{2} \mathrm{Cu}_{1-x} \mathrm{Co}_{x} \mathrm{~F}_{4}$ with $x=0.218 \pm 0.004$, as determined with inductively coupled plasma spectroscopy. The sample was shaped roughly to the form of an ellipsoid with dimensions $7.0 \times 3.9 \times 1.3 \mathrm{~mm}^{3}$ Magnetic moments were measured by use of a vibrating-sample magnetometer with a sensitivity of $10^{-5} \mathrm{emu}$. External fields ranging from $1.0 \mathrm{G}$ to $12.5 \mathrm{kG}$ were applied along the $c$ axis, and the earth magnetic field was compensated. All fields and susceptibilities were corrected for demagnetization. The sample was immersed in liquid helium, the temperature of which was stabilized to within $0.01 \mathrm{~K}$ by controlled pumping. All data of the magnetization $M(T, H)$ were collected under field-cooling conditions, i.e., the field $H$ was switched on at a high temperature $(\approx 30 \mathrm{~K})$, and kept constant during subsequent slow cooling (typically at a rate of $5 \mathrm{mK} / \mathrm{s}$ ) to the temperature of interest.

The results for the static susceptibility $\chi=M / H$ versus the temperature are plotted in Fig. 3 for a selection of fields between $1.0 \mathrm{G}$ and $10 \mathrm{kG}$. It is seen that the susceptibility rises continuously with decreasing temperature through the freezing temperature, which at the time scale

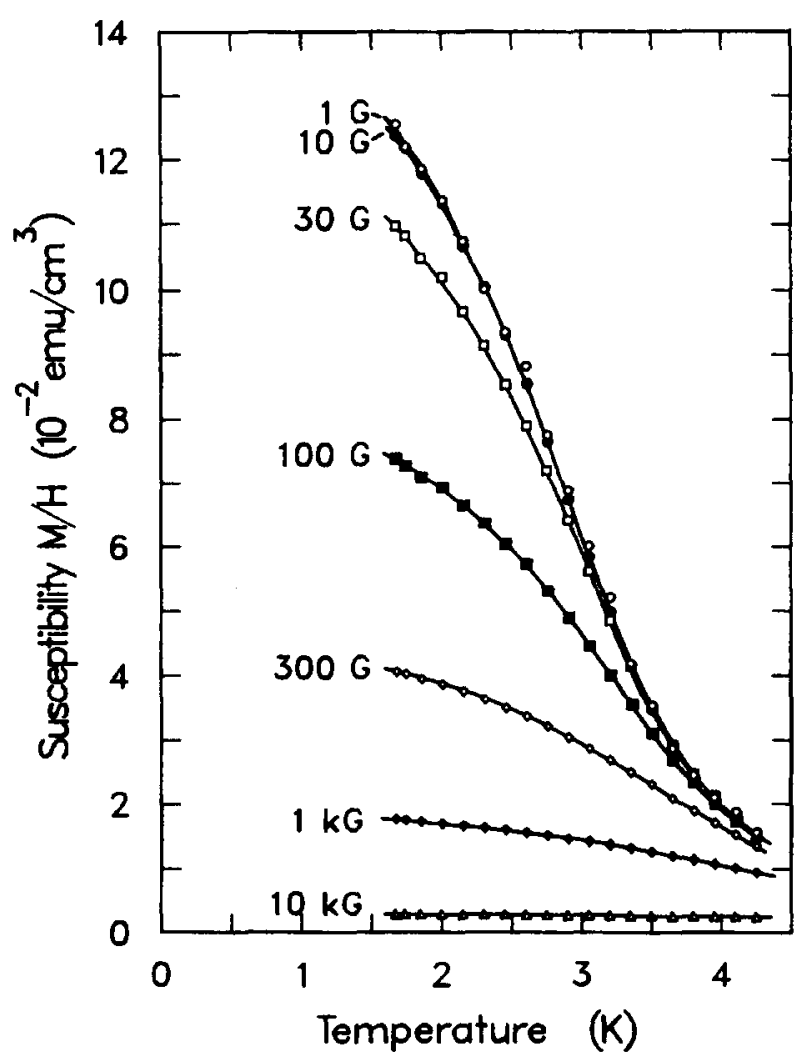

FIG. 3. Static. susceptibility $M / H$ vs the temperature for a selection of external magnetic fields. Solid lines are guides to the eye. typical of the present experiment $\left(\sim 10^{2}\right.$ s) amounts to $T_{f} \approx 2.97 \mathrm{~K}^{27}$ Generally for a $d=2 \mathrm{SG}$, the susceptibility, when observed at a finite time scale, is anticipated to reach a plateau below $T_{f}$ (cf. results of Monte Carlo simulations ${ }^{11}$ ), despite the fact that the equilibrium linear susceptibility keeps rising with decreasing temperature. That such a plateau does not occur in $\mathrm{Rb}_{2} \mathrm{Cu}_{1-x} \mathrm{Co}_{x} \mathrm{~F}_{4}$ is plausible on the grounds that, even in a frozen domain structure, the thermal decrements of the magnetizations residing at individual ions, in particular those of the abundant $\mathrm{Cu}$ ions, have already become significant at temperatures as low as a few Kelvin. Nonequilibrium of the system below approximately $T_{f}$ is manifest from a cooling-rate dependence of the susceptibility in this temperature range. The susceptibility is observed to be quite large, as expected for a concentrated SG at these low temperatures. The susceptibility is further enhanced by residual short-range ferromagnetic correlations, which are apparent from data taken at higher temperatures. In fact, up to about $20 \mathrm{~K}$ the susceptibility follows an effective Curie-Weiss law, i.e., $\chi(T)=C /(T-\theta)$, with $\theta=5.1 \pm 0.2 \mathrm{~K} .^{28}$

The prominent feature contained in the data of Fig. 3 is that the magnetization becomes increasingly nonlinear with the field upon lowering the temperature. In order to examine this in detail, we have measured $M(T, H)$ at a set of discrete temperatures for as many as 35 fields ranging from $1.0 \mathrm{G}$ to $12.5 \mathrm{kG}$. From these data, $M(H)$ isotherms were deduced.

\section{NONLINEAR-SUSCEPTIBILITY ANALYSIS}

In the presentation of the $M(H)$ isotherms, we eliminate the linear part $\chi_{0}$ of the susceptibility in order to find the dimensionless nonlinear part

$$
\chi_{\mathrm{n} 1}(T, H) \equiv 1-\frac{M}{\chi_{0} H}
$$

Here, $\chi_{0}$, the susceptibility in the linear regime, is inserted as deduced from the experiments in small fields. The results for $\chi_{\mathrm{n} 1}(H)$, given in Fig. 4 for a selection of temperatures, clearly manifest a dramatic increase of the nonlinearity with decreasing temperature. To parametrize this, we start from the common $T>T_{c}$ expansion of the magnetization in odd powers of the field,

$$
M=a_{1}\left(\frac{H}{T}\right)-a_{3}\left(\frac{H}{T}\right)^{3}+a_{5}\left(\frac{H}{T}\right)^{5}-a_{7}\left(\frac{H}{T}\right)^{7}+\cdots,
$$

defined such that for a paramagnet the coefficients $a_{1}, a_{3}, \ldots$ are constants. In the case of a SG, however, $a_{3}, a_{5}, \ldots$ are related to the SG order-parameter susceptibility, which exhibits conventional critical behavior at $T_{c} \cdot{ }^{4,5}$ Note that the linear part of the susceptibility $a_{1} / T$, remains nonsingular at $T_{c}$. From expansion of the free energy, ${ }^{4,7}$ it appears that $a_{2 n+1}$ diverges according to $\left(T-T_{c}\right)^{-n(\gamma+\beta)+\beta}$ for $n \geq 1$, i.e., 


$$
\begin{aligned}
& a_{3} \propto\left(T-T_{c}\right)^{-\gamma}, \\
& a_{5} \propto\left(T-T_{c}\right)^{-(2 \gamma+\beta)},
\end{aligned}
$$

An examination of the temperature dependences of $a_{3}, a_{5}, \ldots$ thus allows to determine $T_{c}$ as well as the critical exponents $\gamma$ and $\beta$.

Prior to carrying out this analysis, we have to deal, in one way or another, with the problem that in a real SG the interactions do not perfectly average out to zero, as opposed to Eq. (5), where $\chi_{0}$ is assumed to obey the simple Curie law $\chi_{0} \propto 1 / T$ appropriate to a symmetric distribution of exchange interactions. As is commonly done, we resort to the intuitive procedure, introduced by Omari et al., ${ }^{7}$ of expanding $M$ in powers of $\chi_{0} H$ in place of $H / T$. Equation (5) then becomes

$$
M=\chi_{0} H-a_{3}\left(\chi_{0} H\right)^{3}+a_{5}\left(\chi_{0} H\right)^{5}-a_{7}\left(\chi_{0} H\right)^{7}+\cdots,
$$

or, equivalently,

$$
\chi_{\mathrm{n} 1}=a_{3}\left(\chi_{0} H\right)^{2}-a_{5}\left(\chi_{0} H\right)^{4}+a_{7}\left(\chi_{0} H\right)^{6}-\cdots .
$$

It has recently been argued on the basis of an effectivemean-field model ${ }^{29}$ that this approach, although not entirely rigorous in the case of SG, yields the dominant correction.

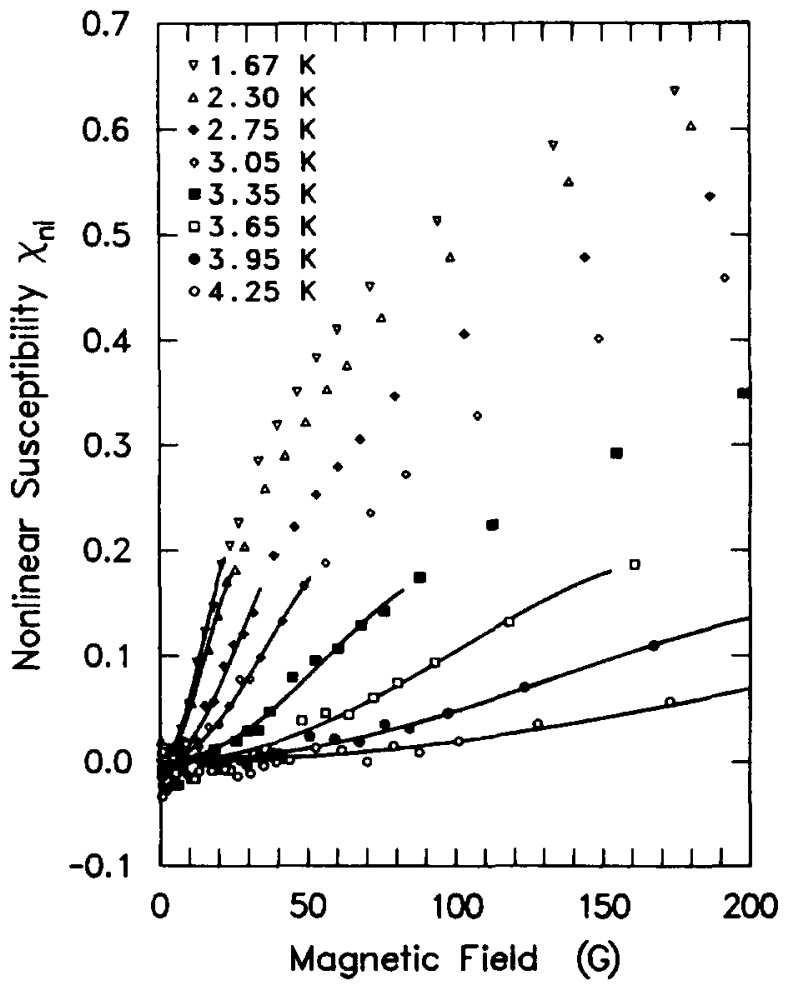

FIG. 4. Nonlinear susceptibility $\chi_{n l}=1-M / \chi_{0} H$ vs the internal field for a selection of temperatures. Solid lines are according to Eq. (8).
Equation (7), cut off after the first three terms, has been fitted to the $M(H)$ isotherms with $\chi_{0}, a_{3}$, and $a_{5}$ as adjustable parameters. The fitting has been restricted to the range of fields where $a_{7}\left(\chi_{0} H\right)^{7}$ is still negligible. Fits of good quality $\left(\chi^{2} \approx 1\right)$ are then found, as is apparent from the solid lines in Fig. 4, which represent Eq. (8) with the resultant fitting parameters inserted. The output values for $\chi_{0}$ and $a_{3}$ further appear to coincide within errors with the results from the fits of Eq. (7) limited to the first two terms. As regards the result for $\chi_{0}$, its temperature dependence essentially equals that of $M / H$ given in Fig. 3 for the fields up to $10 \mathrm{G}$. The resultant temperature dependences of $a_{3}$ and $a_{5}$ are presented in Fig. 5. The rapid increase of both these coefficients is seen to level off at a point somewhat below $T_{f}$, obviously because the slow but finite cooling rate inhibits their equilibrium values to be attained.

Above approximately $T_{f}$, the results for $a_{3}$ and $a_{5}$ are both well accounted for by a power law "series" diverging at $T_{c}=0 \mathrm{~K}$ (solid lines in Fig. 5). This, in fact, comprises a major conclusion of this work. The exponents obtained from fitting of Eqs. (6) are $\gamma=4.5 \pm 0.2$ and $2 \gamma+\beta=9.0 \pm 0.5$. Combination of these results in turn yields $\beta=0.0 \pm 0.7$. In Sec. VI, we will compare the exponents with various theoretical estimates. We note that additional data above $4.25 \mathrm{~K}$ (not presented here),

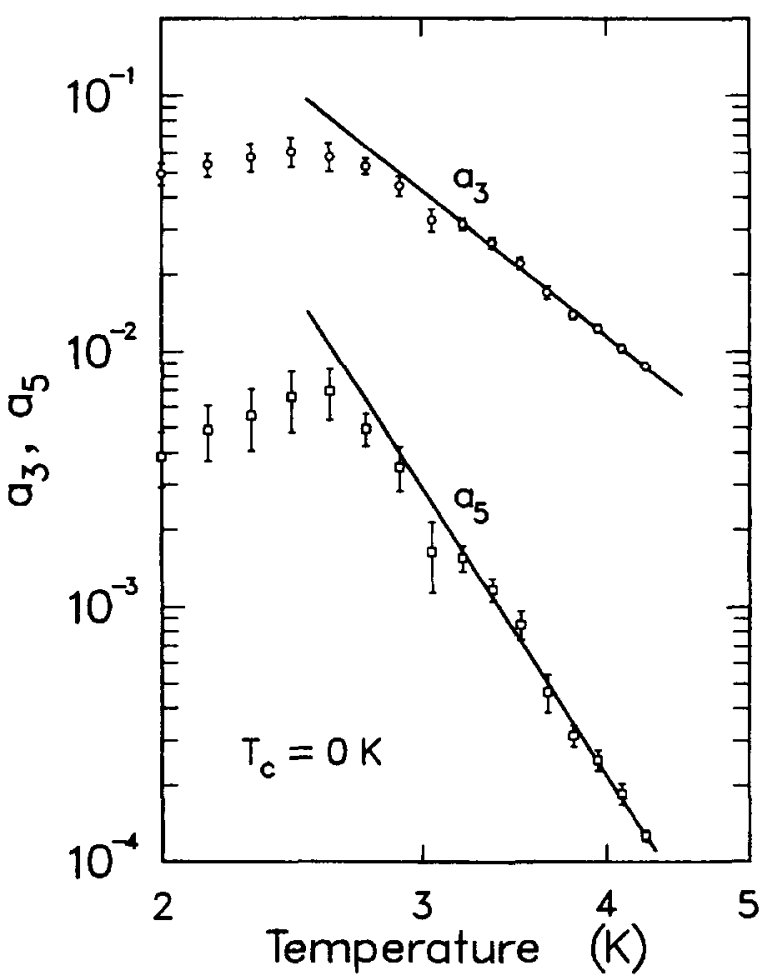

FIG. 5. Nonlinear susceptibility coefficients $a_{3}$ and $a_{5}$ (in emu) vs the temperature. Solid lines denote power-law divergences towards $T_{c}=0 \mathrm{~K}$, Eqs. (6). 


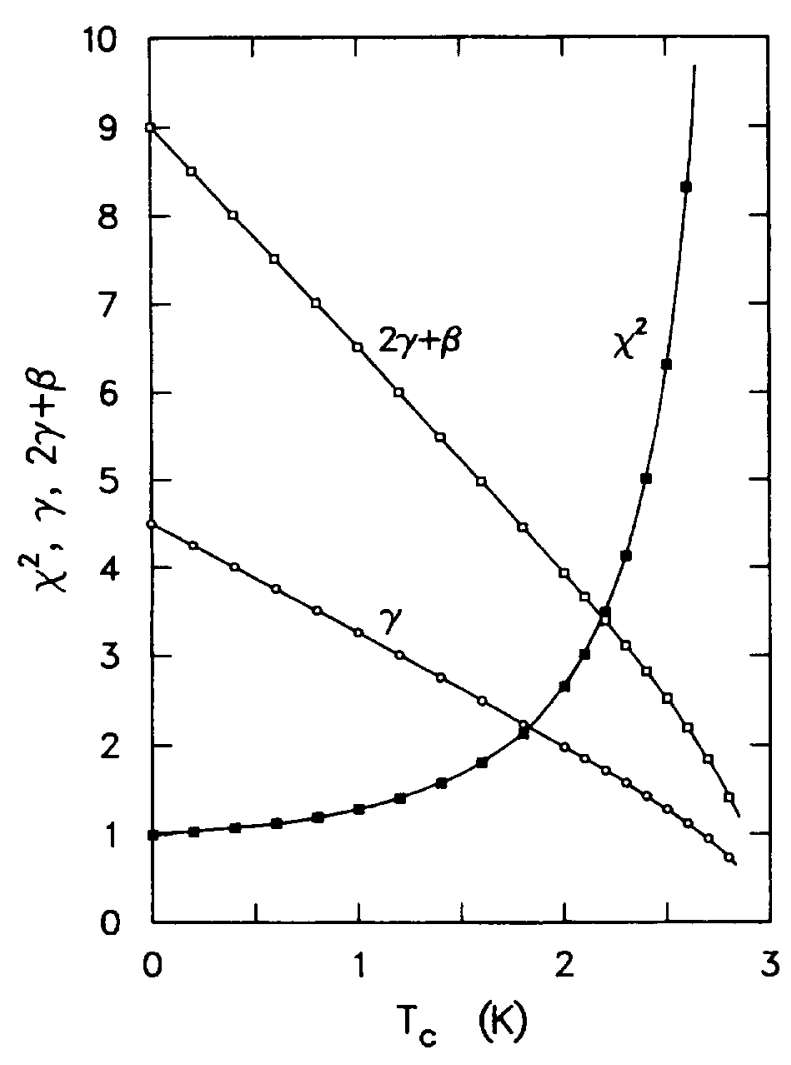

FIG. 6. Results for $\chi^{2}, \gamma$, and $2 \gamma+\beta$ vs $T_{c}$, obtained from fitting Eqs. (6) with a finite $T_{c}$ inserted. Solid lines are guides to the eye.

which suffer from increased scatter, are consistent with the power-law dependences found for $a_{3}$ and $a_{5}$.

In order to establish more firmly that the divergence of the nonlinear susceptibility conforms to $T_{c}=0$, it is expedient to show that a finite $T_{c}$ of order $T_{f}$ is not reconcilable with the experimental findings. To this end, we have additionally adjusted Eqs. (6) to the results for $a_{3}$ and $a_{5}$ above $T_{f}$, but with $T_{c}$ fixed at values ranging from 0 to $T_{f}$. The results for $\gamma$ and $2 \gamma+\beta$, as well as the corresponding $\chi^{2}$, are given in Fig. 6 as a function of $T_{c}$. For values of $T_{c}$ just below $T_{f}(2.5-2.9 \mathrm{~K})$, the fits turn out to be markedly inferior $\left(\chi^{2}=5-40\right)$, while $\chi^{2}$ becomes minimum at $T_{c}=0 \mathrm{~K}$. The results of Fig. 6 thus rule out $T_{c} \approx T_{f}$, but, of course, would be consistent with any $T_{c}$ below about $1 \mathrm{~K}$, such as could be due to residual interlayer coupling. Since the critical behavior is accessible to experiment at feasible time scales only above approximately $3 \mathrm{~K}$, such a small $T_{c}$ would however not detract from $\mathrm{Rb}_{2} \mathrm{Cu}_{1-x} \mathrm{Co}_{x} \mathrm{~F}_{4}$ to realize the $d=2$ SG with $T_{c}=0$.

\section{STATIC SCALING ANALYSIS}

The dependence of the nonlinear susceptibility on the field and the temperature has in addition been examined in the context of static scaling, an approach that has flourished in the field of SG. ${ }^{6-8,30-32}$ Within the scaling picture, all critical physical quantities are determined by the correlation length $\xi$. The underlying ansatz is that in the critical regime the dependences of $\xi$ on $H$ and $T$ can be cast into a scaling form, the relevant argument being $H$ scaled to some power of the temperature. That is, for $T_{c}=0,{ }^{33}$

$$
\xi=T^{-v} f\left(\frac{H}{T^{\Delta}}\right)=H^{-v / \Delta} f\left(\frac{T}{H^{1 / \Delta}}\right),
$$

with $f$ and $f$ scaling functions, and $v$ and $\Delta=1+\frac{1}{2}(\gamma+\beta)$ critical exponents. Note that this scaling relation implies $\xi \propto T^{-v}$ for $H=0$, and $\xi \propto H^{-v / \Delta}$ for $T=0$.

Similarly to $\mathrm{Eq}$. (9), the scaling relation for the nonlinear susceptibility reads $\chi_{\mathrm{nl}}=T^{\beta} \mathrm{g}\left(H / T^{\Delta}\right)^{4,6}$ In order to account for deviations of $\chi_{0}$ from strictly Curie behavior, we replace, as in Sec. IV, $H / T$ by $\chi_{0} H$, to arrive at

$$
\chi_{\mathrm{nl}}=T^{\beta} \mathcal{F}\left(\frac{\chi_{0} H}{T^{\Delta-1}}\right) .
$$

In the limit $x \rightarrow 0$ the scaling function $\mathcal{F}(x)$ is given by $c_{0} x^{2}$, while for $x \rightarrow \infty \mathscr{F}(x)=1-c_{\infty} x^{-1 / \Delta}$, with $c_{0}$ and $c_{\infty}$ constants.

In Fig. 7, the nonlinear susceptibility of $\mathrm{Rb}_{2} \mathrm{Cu}_{0.782} \mathrm{Co}_{0.218} \mathrm{~F}_{4}$ scaled according to Eq. (10), i.e., $\chi_{\mathrm{nl}} T^{-\beta}$ versus $\chi_{0} H / T^{\Delta-1}$, is shown for the entire range

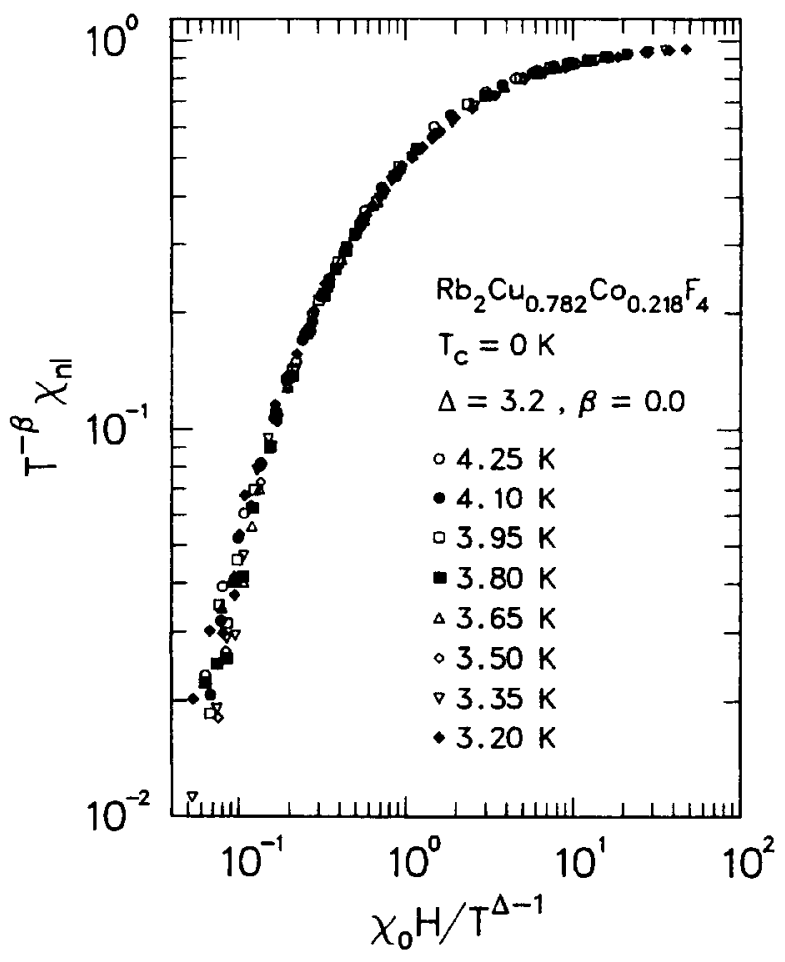

FIG. 7. $T_{c}=0$ scaling plot of the nonlinear-susceptibility data. External fields range from $1.0 \mathrm{G}$ to $12.5 \mathrm{kG}$. 
of fields considered ( $1.0 \mathrm{G}$ to $12.5 \mathrm{kG})$ and temperatures from 4.25 to $3.20 \mathrm{~K}$. It is seen that truly excellent conformity with the $T_{c}=0$ scaling prediction is achieved after adjustment of $\Delta$ and $\beta$ for optimum coincidence of the data on a universal curve. The critical exponents obtained are $\beta=0.0 \pm 0.1$ and $\Delta=3.2 \pm 0.2$, which implies $\gamma=4.4 \pm 0.4$. It has been noticed that critical exponents deduced from scaling may suffer from systematic deviations when using too large a field or temperature range. ${ }^{8}$ In the present case, however, essentially the same results are obtained upon restricting the data set to fields so small that the nonlinear part of $M(H)$ is less than $10 \%$ of $\chi_{0}$. In Sec. VI, the critical exponents will be discussed in some more detail. Inclusion of temperatures below $3.1 \mathrm{~K}$ leads to a deterioration of the fit, obviously because the system has fallen out of equilibrium at these temperatures. The scaling of $\chi_{\mathrm{nl}}$ in $\mathrm{Rb}_{2} \mathrm{Cu}_{0.782} \mathrm{Co}_{0.218} \mathrm{~F}_{4}$ thus further establishes $T_{c}=0$, with essentially the same exponents as derived in the previous section. We gain a more accurate determination of $\beta$, and, more importantly, we are able to extend the analysis to much higher fields. The latter is feasible because Eq. (10) encompasses all singular contributions contained in Eq. (8), whereas the dominant nonlinear terms $a_{3}$ and $a_{5}$ suffice at low fields only.

Our results show an overall agreement with the findings from Monte Carlo simulations of the $d=2$ Edwards-Anderson Ising SG. This includes $T_{c}=0$ scaling of the nonlinear susceptibility and the critical exponents, for which the simulations yielded $\beta=0$ and $\Delta=3.5 \pm 0.5 .^{11}$ Although ample evidence is available that $T_{c}=0$ for the $d=2$ Edwards-Anderson SG model, a remarkable feature of the Monte Carlo simulation data is that they could equally well be scaled to a $d=3$ scaling expression, distinguished from $d=2$ by a nonzero transition temperature. To show that the present data for $\mathrm{Rb}_{2} \mathrm{Cu}_{0.782} \mathrm{Co}_{0.218} \mathrm{~F}_{4}$ exhibit an entirely similar behavior, we have fitted them to the $d=3$ analogue of Eq. (10),

$$
\chi_{\mathrm{n} 1}=t^{\beta} g\left(\frac{\chi_{0} H}{t^{(\gamma+\beta) / 2}}\right],
$$

in which $t=\left(T-T_{c}\right) / T$. The scaling function $S(x)$ behaves as $c_{0} x^{2}$ for small $x$, and as $c_{\infty} x^{2 / 8}$ in the high $x$ limit, with $\delta$ an additional critical exponent. For the range of $T_{c}$ from 3.0 down to $0.5 \mathrm{~K}$, fits of a quality more or less comparable to that of the fit of Eq. (10) are obtained. We find $\delta=21.0 \pm 0.2$, and, with decreasing $T_{c}, \gamma$ varying from 0.8 to 23 , and $\beta$ from 0.04 to 1.1. As in the previous section (cf. Fig. 6), there appears to be a definite trend, albeit a small one, towards better fits with lower $T_{c}$, which is indicative of $T_{c}=0 \mathrm{~K}$.

\section{DISCUSSION AND CONCLUSIONS}

We have successfully examined the nonlinear part of the susceptibility of $\mathrm{Rb}_{2} \mathrm{Cu}_{0.782} \mathrm{Co}_{0.218} \mathrm{~F}_{4}$ on the basis of (i) the power-law divergences of $a_{3}(T)$ and $a_{5}(T)$ towards $T_{c}=0$ and (ii) a static $T_{c}=0$ scaling analysis. This verifies the theoretical evidence for a vanishing $T_{c}$, as obtained for $d=2$ SG models with different random-bond distributions. The two analyses have yielded essentially equal exponents. Summing up, we find $\beta=0.0 \pm 0.1$ and $\gamma=4.5 \pm 0.2$. The result for $\gamma$ agrees within errors with the result recently obtained from dynamic scaling of the ac susceptibility, $\gamma=4.2 \pm 0.6 .^{34}$

In discussing the exponents, we first note that the theoretical results seem to differ for the two best-studied Ising square-lattice SG model systems, viz., the Gaussian and the $\pm J$ model. For the Gaussian model $\beta=0$, while various methods of calculation have resulted in the remarkably high value of $\gamma \approx 7 .^{15}$ Early work on the $\pm J$ model, involving large-scale Monte Carlo simulations and transfer-matrix methods, yielded $\gamma \approx 4 .^{10}$ More recently, a promising new replica Monte Carlo method permitted lower temperatures to be achieved, resulting in $\gamma=5.3 .^{12}$ The same value, $5.3 \pm 0.3$, was found from hightemperature series expansions, ${ }^{17}$ while a finite-size scaling study gave $\gamma=4.5 \pm 0.5 .^{15}$ As regards $\beta$ in the case of the $\pm J$ model, a very small, but finite, value may be estimated from the hyperscaling relation $2 \beta=v(d-2+\eta) .{ }^{15}$ Upon comparing the theoretical results with the present experimental ones, we see that our result for $\gamma, 4.5 \pm 0.2$, seems to be somewhat smaller than the mean of the more recent theoretical estimates for the $d=2 \pm J$ model. The agreement must nevertheless be considered quite satisfactory.

Our results have been derived in a temperature range corresponding to about 0.1 or 0.2 times $\Delta J$, with $\Delta J$ characterizing the width of the bond distribution. For comparison, the results from the Monte Carlo simulations and finite-size scaling pertain to temperatures down to $\sim 0.5 \Delta J$. The present $\gamma$, therefore, is more sure to refer to the true asymptotic critical regime. We further note that the error given for $\gamma$ may be underestimated somewhat because of possible systematic uncertainties contained in the correction for the residual ferromagnetic correlations. To assess the effects of the latter, it would be desirable to similarly determine $\gamma$ in a $\mathrm{Rb}_{2} \mathrm{Cu}_{1-x} \mathrm{Co}_{x} \mathrm{~F}_{4} \mathrm{SG}$ with larger $x$, say $x=0.35$.

In summary, the scaling and the power-law divergence of the static nonlinear susceptibility evidence that the SG transition in the $d=2$ Ising $S G$ model compound $\mathrm{Rb}_{2} \mathrm{Cu}_{1-x} \mathrm{Co}_{x} \mathrm{~F}_{4}$ occurs at zero temperature, with the critical exponents in conformity with theory. Experimental verification is thus provided that the lower critical dimensionality for short-range Ising SG exceeds 2 .

\section{ACKNOWLEDGMENT}

The authors are grateful to M. P. van Eekhout for competent assistance in the experiments.
'K. Binder and A. P. Young, Rev. Mod. Phys. 58, 801 (1986).

${ }^{2}$ Heidelberg Colloquium on Glassy Dynamics, Vol. 275 of Lecture Notes in Physics, edited by J. L. Van Hemmen and 1. Morgenstern (Springer, Heidelberg, 1987).
${ }^{3}$ D. Sherrington and S. Kirkpatrick, Phys. Rev. Lett. 35, 1792 (1975); Phys. Rev. B 17, 4385 (1987).

${ }^{4}$ M. Suzuki, Prog. Theor. Phys. 58, 1151 (1977).

${ }^{5}$ J. Chalupa, Solid State Commun. 22, 315 (1977). 
${ }^{6}$ B. Barbara, A. P. Malozemoff, and Y. Imry, Phys. Rev. Lett. 47, 1852 (1981); B. Barbara and A. P. Malozemoff, J. Less Common Metals 94, 45 (1983), and references therein.

${ }^{7}$ R. Omari, J. J. Prejean, and J. Souletie, J. Phys. (Paris) 44, 1069 (1983).

${ }^{8}$ H. Bouchiat, J. Phys. (Paris) 47, 71 (1986), and references therein.

${ }^{9}$ L. P. Lévy and A. T. Ogielski, Phys. Rev. Lett. 57, 3288 (1986); L. P. Lévy (unpublished).

${ }^{10}$ I. Morgenstern and K. Binder, Phys. Rev. B 22, 288 (1980); A. P. Young, Phys. Rev. Lett. 50, 917 (1980); in Heidelberg Colloquium on Spin Glasses, Vol. 192 of Lecture Notes in Physics, edited by J. L. Van Hemmen and I. Morgenstern (Springer, Heidelberg, 1983), p. 328; W. L. McMillan, Phys. Rev. B 28, 5216 (1983).

${ }^{11}$ W. Kinzel and K. Binder, Phys. Rev. B 29, 1300 (1983); K. Binder and W. Kinzel, in Heidelberg Colloquium on Spin Glasses, Vol. 192 of Lecture Notes in Physics, edited by J. L. Van Hemmen and I. Morgenstern (Springer, Heidelberg, 1983), p. 279; W. Kinzel, in Static Critical Phenomena in Inhomogeneous Systems, Vol. 206 of Lecture Notes in Physics, edited by A. Pȩkalski and J. Sznajd (Springer, Heidelberg, 1984), p. 113.

${ }^{12}$ R. H. Swendsen and J.-S. Wang, Phys. Rev. Lett. 57, 2607 (1986).

${ }^{13}$ H. F. Cheung and W. L. McMillan, J. Phys. C 16, 7027 (1983); 16, 7033 (1983); D. A. Huse and I. Morgenstern, Phys. Rev. B 32, 3032 (1985).

${ }^{14}$ W. L. McMillan, Phys. Rev. B 29, 4026 (1984).

${ }^{15}$ R. N. Bhatt and A. P. Young, in Ref. 2, p. 215 , and references therein.

${ }^{16}$ A. J. Bray and M. A. Moore, in Ref. 2, p. 121, and references therein.

${ }^{17}$ R. R. P. Singh and S. Chakravarty, Phys. Rev. Lett. 57, 245 (1986); Phys. Rev. B 36, 559 (1987).

${ }^{18}$ C. Dekker, A. F. M. Arts, and H. W. de Wijn, J. Appl. Phys. 63, 4334 (1988).
${ }^{19}$ S. F. Edwards and P. W. Anderson, J. Phys. F 5, 965 (1975).

${ }^{20}$ E. J. Samuelsen, Phys. Rev. Lett. 31, 936 (1973); J. Phys. Chem. Solids 35, 785 (1974).

${ }^{21}$ M. T. Hutchings, H. Ikeda, and E. Janke, Phys. Rev. Lett. 49, 386 (1982).

${ }^{22}$ C. Dekker, A. F. M. Arts, and H. W. de Wijn, Phys. Rev. B (to be published).

${ }^{23}$ D. I. Komskii and K. I. Kugel, Solid State Commun. 13, 763 (1973).

${ }^{24} \mathrm{P}$. W. Anderson, in Magnetism, edited by G. T. Rado and $\mathbf{H}$. Suhl (Academic, New York, 1963), Vol. I, p. 25.

${ }^{25}$ L. Onsager, Phys. Rev. 65, 117 (1944).

${ }^{26}$ I. Yamada, J. Phys. Soc. Jpn. 33, 979 (1972).

${ }^{27} T_{f}$ is strongly time dependent, i.e., varying by roughly $0.1 \mathrm{~K}$ per decade in time. The value of $2.97 \mathrm{~K}$ quoted above is as found from the cusp in the zero-field-cooled susceptibility (Refs. 18 and 22).

${ }^{28}$ Above $20 \mathrm{~K}$, again a Curie-Weiss temperature dependence can be discerned, but with a much lower $\theta$, indicating that the overall departure from a distribution of interactions symmetric about zero is much less severe than suggested by the above effective $\theta=5.1 \mathrm{~K}$.

${ }^{29}$ I. Yeung, R. M. Rosko, and G. Williams, J. Magn. Magn. Mater. 68, 39 (1987).

${ }^{30}$ C. Pappa, J. Hammann, and C. Jacoboni, J. Phys. (Paris) 46, 637 (1985).

${ }^{31}$ P. Svedlindh, L. Lundgren, P. Nordblad, and H. S. Chen, Europhys. Lett. 2, 805 (1986).

${ }^{32}$ E. Vincent and J. Hammann, J. Phys. C 20, 2659 (1987).

${ }^{33}$ The scaling form treated here applies to a zero-temperature transition. For a transition at finite $T_{c}, T$ should be replaced by a reduced temperature [cf. Eq. (11)]. The notation is kept consistent with the one of Kinzel and Binder (Ref. 11) in their Monte Carlo simulation study of the $d=2$ Ising SG.

${ }^{34}$ C. Dekker, A. F. M. Arts, H. W. de Wijn, A. J. van Duyneveld, and J. A. Mydosh, Phys. Rev. Lett. 61, 1780 (1988). 